\title{
NONLINEAR VOLTERRA FUNCTIONAL EQUATIONS AND LINEAR PARABOLIC DIFFERENTIAL SYSTEMS $\left({ }^{1}\right)$
}

\author{
BY \\ J. YEH
}

1. Introduction. In his recent paper [1](2) Professor R. H. Cameron pointed out that there exists a certain duality between almost everywhere $\left({ }^{3}\right)$ type existence problems for solutions of nonlinear Volterra functional equations and minimality problems of positive solutions for certain linear parabolic differential systems. At the conclusion of that paper he stated that he expected to follow it with another paper where the duality principle is put to work and that he expected to prove at least one theorem of each type without using the duality principle, and then prove an equal number of corresponding theorems of opposite types by using the duality principle. The nonlinear Volterra functional equations that were studied were, for the two-dimensional case, functional equations of the type

$$
y(t)=x(t)+\int_{0}^{t} F^{2}\left\{s, x(s), \int_{0}^{s} F^{1}[r, x(r)] d r\right\} d s .
$$

The definition of the functional equations in the general $n$-dimensional case will be given in $\$ 2$. The related linear parabolic differential systems were, in the general $n$-dimensional case,

$$
\begin{gathered}
\frac{1}{4} \frac{\partial^{2} \theta}{\partial u^{2}}-\frac{\partial \theta}{\partial t}+J^{1}(t, u) \frac{\partial \theta}{\partial v_{1}}+J^{2}\left(t, u, v_{1}\right) \frac{\partial \theta}{\partial v_{2}}+\cdots \\
+J^{n-1}\left(t, u, v_{1}, \cdots, v_{n-2}\right) \frac{\partial \theta}{\partial v_{n-1}} \\
\quad+J^{n}\left(t, u, v_{1}, \cdots, v_{n-1}\right) \theta=0 \\
\theta\left(0, u, v_{1}, \cdots, v_{n-1}\right)=L\left(u, v_{1}, \cdots, v_{n-1}\right) .
\end{gathered}
$$

The task of proving theorems of the above mentioned nature was then handed over to the present author. Since then two everywhere-type existence

Received by the editors March 19, 1959 and, in revised form, July 10, 1959.

(1) This research was supported in part by the United States Air Force, through the Office of Scientific Research of the Air Research and Development Command under contract No. AF 18(603)-30. The author wishes to thank Professor R. H. Cameron for his valuable suggestions in the writing of this paper.

(2) Numbers in square brackets refer to bibliography.

(3) "Almost everywhere" means all except for a set of Wiener measure zero. See for instance [2]. In this paper Wiener measure and integral will be denoted by $m_{w}$ and $\int \cdots d_{w} x$ respectively. 
theorems, namely, Theorems I, II, and then by applying the duality principle on them, three corresponding minimality theorems, namely Theorems III, IV, $\mathrm{V}$ have been proved. On the other hand a uniqueness theorem for solutions of the differential system, namely Theorem VI, has been found.

The proofs of these theorems constitute this paper. Theorems I, II were derived by classical methods in the sense that no knowledge of Wiener measure was assumed in the proof. Theorems III, IV, V were derived from Theorems I, II by means of Theorems 1, 4, 6 of [1]. Theorem VI is based on Theorem 8 of [1].

2. The general Volterra functional. Throughout this paper the letter $I$ denotes the interval $0 \leqq t \leqq 1, I_{0}$ the interval $0 \leqq t \leqq t_{0}, R_{n}$ the $n$-dimensional Euclidean space. The symbol $C_{w}[a, b]$ denotes the Wiener space on the interval $a \leqq t \leqq b$, namely the space of continuous functions on $a \leqq t \leqq b$ which vanish at $t=a$. In particular $C_{w}[0,1]$ is often abbreviated as $C_{w}$. In order to shorten the notation for functions and functionals of several variables, we adopt the following notational convention which was introduced in [1].

Notation. When a functional depends on a function and several numerical variables, the function will be written first, followed by a bar. The notation for functionals and also for functions may be abbreviated by dropping the function or some or all of the numerical variables, but we stipulate that if the function is dropped, the bar must also be dropped, and if a numerical variable is dropped, all those that follow it must also be dropped. Moreover, whenever a function or variable is dropped, it is understood that the suppressed arguments are the letters which originally stood in the function or functional when it was first introduced, with no substitutions. We also stipulate that if a numerical variable is replaced by some other letter, or by a number it will not be suppressed and none of the numerical variables preceding it will be suppressed.

Thus, for example, the functional $\Psi\left[x(\cdot) \mid t, u, v_{1}, \cdots, v_{n}\right]$ could be abbreviated as $\Psi, \Psi(x \mid), \Psi(t), \Psi(t, u), \Psi\left(x \mid t, u, v_{1}\right)$ etc. but could not be abbreviated as $\Psi(x), \Psi(u), \Psi\left(u, v_{1}, v_{3}\right)$ etc. The symbol $\Psi(0)$ means that $t$ has been replaced by zero while other arguments are left alone, $\Psi(u)$ would mean that $t$ has been replaced by $u$ and would be the same as $\Psi(u, u)$.

With the above notational convention, we now give our formal definition of the Volterra functional.

Definition. Consider a finite sequence of real continuous functions $F^{1}(t, u), F^{2}\left(t, u, v_{1}\right), \cdots, F^{n}\left(t, u, v_{1}, \cdots, v_{n-1}\right)$ defined for $t \in I$ and other variables unrestricted. From these construct inductively two sequences of functionals $\Phi^{k}(x \mid t), \Lambda^{k}(x \mid t)$ depending on the function $x(\cdot)$ and the real variable $t$ as follows:

$$
\begin{array}{lr}
\Lambda^{0}(x \mid t)=x(t), & \text { on } C_{w} \otimes I, \\
\Phi^{k}(x \mid t)=F^{k}\left(t, \Lambda^{0}, \cdots, \Lambda^{k-1}\right), & (k=1,2, \cdots, n) \text { on } C_{w} \otimes I,
\end{array}
$$




$$
\Lambda^{k}(x \mid t)=\int_{0}^{t} \Phi^{k}(\tau) d \tau
$$

$$
(k=1,2, \cdots, n) \text { on } C_{w} \otimes I .
$$

Then the functionals $\Phi^{k}$ and $\Lambda^{k}$ will be called "Volterra functionals" and $\Phi^{n}$ and $\Lambda^{n}$ will be called Volterra functionals of order $n$ if no shorter string of functions could be used to define them.

Now to come to our problem, let $F^{1}, F^{2}, \cdots, F^{n}$ be defined and continuous on $I \otimes R_{n}$ and consider the Volterra functional equation

$$
y(t)=x(t)+\Lambda^{n}(x \mid t)
$$

or with $f=F^{n}(4)$

$$
y(t)=x(t)+\int_{0}^{t} f\left[s, \Lambda^{0}(x \mid s), \cdots, \Lambda^{n-1}(x \mid s)\right] d s .
$$

Then for any given $x \in C_{w}, y$ defined by (2.3) belongs to $C_{w}$. The question here is under what restrictions on $F^{1}, F^{2}, \cdots, F^{n}$ the equation (2.3) has a solution $x \in C_{w}$ for every or for almost every $y \in C_{w}$. Before we answer this question we quote a uniqueness theorem for solutions of (2.3) in the following remark. For the proof, the reader is referred to $[1$, p. 145].

Remark 1. Let $F^{1}(t, u), F^{2}\left(t, u, v_{1}\right), \cdots, F^{n}\left(t, u, v_{1}, \cdots, v_{n-1}\right)$ and their first derivatives with respect to $u, v_{1}, \cdots, v_{n-1}$ be continuous on $I_{0} \otimes R_{n}$. Then if $x_{1}$ and $x_{2}$ both belong to $C_{w}\left[0, t_{0}\right]$ and satisfy (2.3) for a given $y \in C_{w}\left[0, t_{0}\right], x_{1}=x_{2}$.

3. An everywhere-type existence theorem for solutions of Volterra functional equations.

Theorem I. Let $F^{1}(t, u), F^{2}\left(t, u, v_{1}\right), \cdots, F^{n}\left(t, u, v_{1}, \cdots, v_{n-1}\right)$ and their first derivatives with respect to $u, v_{1}, \cdots, v_{n-1}$ be continuous on $I \otimes R_{k}$, $(k=1,2, \cdots, n)$, and let $F^{1}, F^{2}, \cdots, F^{n}$ satisfy

$$
\left|F^{k}\left(l, u, v_{1}, \cdots, v_{k-1}\right)\right| \leqq A\left\{1+\sum_{j=0}^{k-1}\left|v_{j}\right|\right\} \log \left\{2+\sum_{j=0}^{k-1}\left|v_{j}\right|\right\}
$$

$$
\text { an } I \otimes R_{k},(k=1,2, \cdots, n)\left({ }^{5}\right),
$$

for some positive number $A$. Then for every $y \in C_{w}$ the Volterra functional equation (2.3) has a solution $x \in C_{w}$. Moreover the solution is unique in $C_{w}$.

The proof of Theorem I is based on Lemma 3 and Lemma 4. Lemma 3 is based on Lemma 1 and Lemma 2.

3.1. Lemma 1. Let $F^{1}, F^{2}, \cdots, F^{n}$ be continuous on $I \otimes R_{k}(k=1,2, \cdots, n)$ and absolutely continuous with respect to each of $u, v_{1}, \cdots, v_{n-1}$. Let the first derivatives of $F^{1}, F^{2}, \cdots, F^{n}$ with respect to $u, v_{1}, \cdots, v_{n-1}$ exist everywhere

$\left(^{4}\right)$ Later, in $\$ \S 5-6$, as in [1], the notation $F^{n}$ will mean a function other than the $n$th in the succession $F^{1}, F^{2}, \cdots$. For the latter, the notation $f$ will be used.

(5) We use $v_{0}$ for $u$. 
except on a finite number of hyperplanes of the form $v_{j}=$ const. where the right and the left hand derivatives exist, and satisfy

$$
\begin{array}{r}
\left|F_{j}^{k}\left(t, u, v_{1}, \cdots, v_{k-1}\right)\right| \leqq M_{j}^{k}, \quad(j=0,1,2, \cdots, k-1), \\
(k=1,2, \cdots n),
\end{array}
$$

where the $M$ 's are positive constants and $F_{j}^{k}$ stands for $\partial F^{k} / \partial V_{j}$. Then there exist positive constants $B_{1}, B_{2}, \cdots, B_{n}$ such that for any $x_{1}, x_{2} \in C_{w}$

$$
\begin{aligned}
\left|\Lambda^{k}\left(x_{1} \mid t\right)-\Lambda^{k}\left(x_{2} \mid t\right)\right| \leqq B_{k} \int_{0}^{t}\left|x_{1}(s)-x_{2}(s)\right| d s, \\
\quad \text { for } t \in I,(k=1,2, \cdots, n) .
\end{aligned}
$$

Proof. We remark that if $F(v)$ is an absolutely continuous function of the real variable $v$ and $d F / d v$ exists everywhere except at a finite number of points $v$ where the right and the left hand derivatives exist, and is bounded by $M$, then

$$
\left|F\left(v_{2}\right)-F\left(v_{1}\right)\right| \leqq M\left|v_{2}-v_{1}\right| .
$$

From this remark, (3.3) holds by complete induction on $k$ with $B_{1}=M_{0}^{1}$ and in general $B_{N+1}=M_{0}^{N+1}+M_{1}^{N+1} B_{1}+\cdots+M_{N}^{N+1} B_{N}$ for $N=0,1, \cdots$, $n-1$.

At this point we make the following observation which will be needed later.

Remark 2. Suppose $F^{1}, F^{2}, \cdots, F^{n}$ satisfy the conditions in Lemma 1. Then if for some $y \in C[0,1]$, there exist $x_{1}, x_{2} \in C\left[0, t_{0}\right]$ with $t_{0} \leqq 1$ which satisfy (2.3), it follows that $x_{1}(t)=x_{2}(t)$ for $t \in\left[0, t_{0}\right]$.

Proof. With (3.3), Remark 2 can be proved exactly in the same way as Remark 1.

3.2. Lemma 2. If $F^{1}, F^{2}, \cdots, F^{n}$ are continuous on $I \otimes R_{k}(k=1,2, \cdots, n)$ and $\left\{x_{p}(t)\right\}$ is a sequence of functions which are continuous on $I$ and converges to a function $x(t)$ uniformly on $I$, then

$$
\lim _{p \rightarrow \infty} \Lambda^{k}\left(x_{p} \mid t\right)=\Lambda^{k}(x \mid t) \text { uniformly in t on } I, \quad(k=0,1,2, \cdots, n) .
$$

Proof. From the fact that $F^{k}$ is uniformly continuous on $I \otimes S_{k}$ where $S_{k}$ is a closed rectangle in $R_{k}$, the conclusion of the lemma follows immediately by complete induction on $k$.

3.3. Lemma 3. Let $F^{1}, F^{2}, \cdots, F^{n}$ satisfy the hypothesis of Lemma 1. Then for any $y \in C_{w}$, there exists a unique element $x$ of $C_{w}$ which satisfies (2.3).

Proof. Let $y \in C_{w}$ be given. Let us define a sequence of functions $\left\{x_{p}(t)\right\}$ on $I$ by 


$$
x_{p+1}(t)=y(t)-\Lambda^{n}\left(x_{p} \mid t\right), \quad(p=0,1,2, \cdots)
$$

with $x_{0}=y$. Clearly $x_{p} \in C_{w}$ for $p=0,1,2, \cdots$. By (3.5), (3.3) and an induction on $p$, we obtain

$$
\left|x_{p+1}(t)-x_{p}(t)\right| \leqq \frac{b\left(B_{n} t\right)^{p}}{p !}, \quad \text { for } t \in I, p=0,1,2, \cdots,
$$

where $b=\max \left|F^{n}\right|$ on the bounded closed set $I \otimes I^{(0)} \otimes I^{(1)} \otimes \cdots \otimes I^{(n-1)}$ and

$$
I^{(k)}=\left\{v_{k} \in R_{1} \mid \min _{t \in I} \Lambda^{k}(y \mid t) \leqq v_{k} \leqq \max _{t \in I} \Lambda^{k}(y \mid t)\right\} .
$$

By (3.6) and by Cauchy's criterion, $\left\{x_{p}\right\}$ converges uniformly on $I$ to an element $x$ of $C_{w}$.

This $x$ is a solution of (2.3), for

$$
x(t)=\lim _{x \rightarrow \infty} x_{p}(t)=y(t)-\Lambda^{n}(x \mid t)
$$

by (3.5), (3.4). The uniqueness of this solution is from Remark 2.

3.4. Lemma 4. Let $F^{1}, F^{2}, \cdots, F^{n}$ be continuous on $I \otimes R_{k}(k=1,2, \cdots, n)$ and satisfy (3.1). For a given $x \in C_{w}$, define $y \in C_{w}$ by (2.3). Then there exists $a$ positive number $B$, independent of $x$, such that $\left({ }^{6}\right)$

$$
\left\|\left|\Lambda^{k}(x \mid t)\right|\right\| \leqq B\left\{1+\|y\|^{B}\right\}, \quad(k=1,2, \cdots, n),
$$

where $\|y\|$ is the Hilbert norm of $y$, i.e. $\|x\|=\left\{\int_{0}^{1}[x(t)]^{2} d t\right\}^{1 / 2}$.

Proof. Let $x \in C_{w}$ be given and let $y \in C_{w}$ be defined by (2.3). Let

$$
\begin{aligned}
& w_{0}(t)=y(t)-x(t)=\Lambda^{n}(x \mid t), \\
& w_{k}(t)=\Lambda^{k}(x \mid t),
\end{aligned} \quad(k=1,2, \cdots, n-1) .
$$

Then by (3.8), (2.2), (2.1), (3.1) we have the following estimate for $w_{0}(t)$;

$$
\left|w_{0}(t)\right| \leqq A\left\{Y(t)+\sum_{j=0}^{n-1} W_{j}(t)\right\}
$$

where

$$
\begin{aligned}
& Y(t)=\int_{0}^{t}\{1+(n+1)|y(s)|\} \log \{2+(n+1)|y(s)|\} d s, \\
& W_{k}(t)= \int_{0}^{t}\left\{1+(n+1)\left|w_{k}(s)\right|\right\} \log \left\{2+(n+1)\left|w_{k}(s)\right|\right\} d s, \\
&(k=0,1,2, \cdots, n-1) .
\end{aligned}
$$

(6) We define ||$|x| \|=\max _{t \in I}|x(t)|$ for any function $x(t)$ defined on $t \in I$. 
By (3.11), (3.12), $Y(t), W_{k}(t),(k=0,1,2, \cdots, n-1)$ are positive and monotonically increasing functions of $t$ on $I$. Similarly from (3.9), (2.2), (2.1), (3.1) we obtain

$$
\left|w_{k}(t)\right| \leqq A\left\{Y(t)+\sum_{j=0}^{k-1} W_{j}(t)\right\}, \quad(k=1,2, \cdots, n-1) .
$$

We now proceed to eliminate $Y(t), W_{k}(t)$ from (3.10), (3.13) by estimating first $W_{k}(t)$ by $Y(t)$ and then $Y(t)$ by $\|y\|$. Let $Y_{0}(t) \equiv\|\| Y \mid \|=Y(1)\left({ }^{6}\right)$. Then by (3.12), (3.10), (3.13)

$$
\begin{aligned}
\frac{d W_{k}(t)}{d t} \leqq & \left\{1+(n+1) A\left(Y_{0}+\sum_{j=0}^{n-1} W_{j}(t)\right)\right\} \\
& \cdot \log \left\{2+(n+1) A\left(Y_{0}+\sum_{j=0}^{n-1} W_{j}(t)\right)\right\}
\end{aligned}
$$

for $k=0,1,2, \cdots, n-1$. From the above $n$ inequalities

$$
\begin{aligned}
&(n+1) A \frac{d}{d t}\left\{\sum_{k=0}^{n-1} W_{k}(t)\right\}\left\{2+(n-1) A\left(Y_{0}+\sum_{k=0}^{n-1} W_{k}(t)\right)\right\}^{-1} \\
& \cdot \log \left\{2+(n+1) A\left(Y_{0}+\sum_{k=0}^{n-1} W_{k}(t)\right)\right\}^{-1} \leqq n(n+1) A
\end{aligned}
$$

and upon integrating with respect to $t$

$$
\log \log \left\{2+(n+1) A\left(Y_{0}+\sum_{k=0}^{n-1} W_{k}(s)\right)\right\} \int_{0}^{t} \leqq n(n+1) A t
$$

and then from the fact that $W_{k}(0)=0,(k=0,1,2, \cdots, n-1)$

$$
2+(n+1) A\left(Y_{0}+\sum_{k=0}^{n-1} W_{k}(t)\right) \leqq\left\{2+(n+1) A Y_{0}\right\}^{B_{1}}
$$

with $B_{1}=\exp \{n(n+1) A\}$ and finally

(3.14) $\quad W_{k}(t) \leqq \frac{1}{(n+1) A}\left\{2+(n+1) A Y_{0}\right\}^{B_{1}}, \quad(k=0,1,2, \cdots, n-1)$.

By (3.11) and the fact that $\log (1+u) \leqq u$ for $u \geqq 0$ and $|y(t)| \leqq 1+|y(t)|^{2}$ on $I$,

(3.15) $\quad Y_{0} \leqq \int_{0}^{1}\{1+(n+1)|y(t)|\}^{2} d t \leqq 3(n+1)^{2}\left(1+\|y\|^{2}\right)$,

and from (3.14) 


$$
W_{k}(t) \leqq \frac{1}{(n+1) A}\left\{2+3(n+1)^{3} A\left(1+\|y\|^{2}\right)\right\}^{B_{1}}, \quad(k=0,1,2, \cdots, n-1) .
$$

We may assume without loss of generality that $A \geqq 1$. Then since $n \geqq 1$, it follows that $B_{1}>1$ and

(3.16) $W_{k}(t) \leqq \frac{1}{(n+1) A}\left\{4(n+1)^{3} A\left(1+\|y\|^{2}\right)\right\}^{B_{1}} \quad(k=0,1,2, \cdots, n-1)$.

Then from (3.10), (3.13), (3.15), (3.16)

$$
\begin{array}{r}
\left|w_{k}(t)\right| \leqq 3(n+1)^{2} A\left(1+\|y\|^{2}\right)+\frac{n}{n+1}\left\{4(n+1)^{3} A\right\}^{B_{1}}\left(1+\|y\|^{2}\right)^{B_{1}} \\
(k=0,1,2, \cdots, n-1)
\end{array}
$$

and from the fact that $(n+1)^{3} A>1$ and $B_{1}>1$, it follows that $3(n+1)^{2} A$ $\leqq(n+1)^{-1}\left\{4(n+1)^{3} A\right\}^{B_{1}}$ and hence

$$
\left|w_{k}(t)\right| \leqq\left\{4(n+1)^{3} A\right\}^{B_{1}}\left(1+\|y\|^{2}\right)^{B_{1}}, \quad(k=0,1,2, \cdots, n-1) .
$$

Also from the fact that $B_{1}>1$, we have $\left(1+\|y\|^{2}\right)^{B_{1}} \leqq 2^{B_{1}}\left\{1+\|y\|^{2 B_{1}}\right\}$ so that

$$
\left|w_{k}(t)\right| \leqq\left\{8(n+1)^{3} A\right\}^{B_{1}}\left\{1+\|y\|^{2 B_{1}}\right\}, \quad(k=0,1,2, \cdots, n-1) .
$$

If we let $B=\max \left\{\left\{8(n+1)^{3} A\right\}^{B_{1}}, 2 B_{1}\right\}$, then

$$
\left|w_{k}(t)\right| \leqq B\{1+\|y\|\}^{B}, \quad(k=0,1,2, \cdots, n-1) .
$$

3.5. Proof of Theorem I. Let $y \in C_{w}$ be given and let

$$
S=B(1+\|y\| B)+\|\mid y\|
$$

where $B$ is a positive constant specified in Lemma 4 . Let us define a sequence of functions $F^{1 *}(t, u), F^{2 *}\left(t, u, v_{1}\right), \cdots, F^{n *}\left(t, u, v_{1}, \cdots, v_{n-1}\right)$ by stipulating that for $k=1,2, \cdots, n, F^{k *}$ is defined to be equal to $F^{k}$ if $\left|v_{j}\right| \leqq S$ for all $j=0,1,2, \cdots, k-1$, and if $v_{j_{0}}>S$ or $v_{j_{0}}<-S, \cdots, v_{j_{m}}>S$ or $v_{j_{m}}<-S$ where $m$ is any of $0,1,2, \cdots, k-1, F^{k *}$ is defined to be equal to the value of $F^{k}\left(t, u, v_{1}, \cdots, v_{k-1}\right)$ with $v_{j_{0}}, \cdots, v_{j_{m}}$ replaced by $S$ or $-S$. Then $F^{* *},(k=1,2, \cdots, n)$, satisfy the hypotheses of Lemma 3 and hence there exists $x \in C_{w}$ such that

$$
y(t)=x(t)+\Lambda^{n *}(x \mid t)
$$

where $\Lambda^{k *}(x \mid t)(k=0,1,2, \cdots, n)$ are defined by (2.0), (2.1), (2.2) with $F^{* *}(k=0,1,2, \cdots, n)$. By Lemma 4 and (3.17)

$$
\|x\| \leqq S, \quad\left\|\left|\Lambda^{k *}(x \mid t)\right|\right\| \leqq S, \quad(k=1,2, \cdots, n-1) .
$$

Now since $F^{k}$ are identical with $F^{* *}$ on $\left|v_{j}\right| \leqq S$ for $j=0,1,2, \cdots, k-1$ and $k=1,2, \cdots, n, \Lambda^{k *}(x \mid t)=\Lambda^{k}(x \mid t)$ for $t \in I$ and $k=0,1,2, \cdots$, for our $x$. 
Thus our $x$ satisfies (2.3). The uniqueness of this solution $x$ follows by Remark 1.

4. Another everywhere-type existence theorem for solutions of Volterra functional equations.

TheOREM II. Let $F^{1}(t, u), F^{2}\left(t, u, v_{1}\right), \cdots, F^{n}\left(t, u, v_{1}, \cdots, v_{n-1}\right)$ and their first derivatives with respect to $u, v_{1}, \cdots, v_{n-1}$ be continuous on $I \otimes R_{k}(k=1,2, \cdots, n)$ and let $F^{n}$ satisfy the condition

$$
F^{n}\left(t, u, v_{1}, \cdots, v_{n-1}\right) \operatorname{sgn} u \geqq-A
$$

an $I \otimes R_{n}$

where $A$ is a constant. Then the Volterra functional equation (2.3) has a solution $x \in C_{w}$ for every $y \in C_{w}$. Moreover the solution $x$ is unique in $C_{w}$.

Theorem II is based on Lemma 7 which is based on Lemmas 5 and 6 .

4.1. Lemma 5. Let $F^{1}, F^{2}, \cdots$ together with their first derivatives with respect to $u, v_{1}, v_{2}, \cdots$ be continuius on $I \otimes R_{k}(k=1,2, \cdots)$. Let $a$ and $b$ satisfy $0 \leqq a \leqq b \leqq 1$, let $\xi(t)$ be an arbitrary continuous function defined on $0 \leqq t \leqq a$ and let $U$ be an arbitrary constant satisfying $U \geqq|\xi(a)|$. Let $\mathfrak{M}(a, b, \xi, U)$ be the totality of functions $x(t)$ defined and continuous on $0 \leqq t \leqq b$ and satisfying

$$
\begin{aligned}
x(t) & =\xi(t) & & \text { on } 0 \leqq t \leqq a, \\
|x(t)| & \leqq U & & \text { on } a \leqq t \leqq b .
\end{aligned}
$$

Then there exists a sequence of constants $B_{1}, B_{2}, \cdots$ such that

$$
\left|\Lambda^{k}\left(x_{2} \mid t\right)-\Lambda^{k}\left(x_{1} \mid t\right)\right| \leqq B_{k} \int_{a}^{t}\left|x_{2}(s)-x_{1}(s)\right| d s, \quad(k=1,2, \cdots)
$$

hold for all $t$ in $a \leqq t \leqq b$ and any pair of elements $x_{1}, x_{2}$ of $\mathfrak{M}$.

We remark that the conclusion of Lemma 5 follows by complete induction on $k$ using boundedness of $\partial F^{k} / \partial v_{j}$.

REmark 3. Consider $\mathfrak{M}\left(a, b_{1}, \xi, U\right)$ and $\mathfrak{M}\left(a, b_{2}, \xi, U\right)$ with $0 \leqq a \leqq b_{1} \leqq b_{2}$ $\leqq 1$. If $B_{1}, B_{2}, \cdots$ is a sequence of constants with which (4.4) holds for all $t$ in $a \leqq t \leqq b_{2}$ for any $x_{1}, x_{2} \in \mathfrak{M}\left(a, b_{2}, \xi, U\right)$, then (4.4) holds with this sequence for all $t$ in $a \leqq t \leqq b_{1}$ for any $x_{1}, x_{2} \in \mathfrak{M}\left(a, b_{1}, \xi, U\right)$.

4.2. Lemma 6. Let $F^{1}, F^{2}, \cdots, F^{n}$ and their first derivatives with respect to $u, v_{1}, \cdots, v_{n-1}$ be continuous on $I \otimes R_{k}(k=1,2, \cdots, n)$. Let a satisfy $0 \leqq a<1$ and let $\xi(t)$ be an arbitrary function defined and continuous on $0 \leqq t \leqq a$. Then for any function $\eta(t)$ defined and continuous on $a \leqq t \leqq 1$, there exists a function $x(t)$ which is continuous on $0 \leqq t \leqq b$ for some $b$ such that $a<b \leqq 1$ and satisfies

$$
\begin{aligned}
x(t) & =\xi(t) \\
\eta(t)-\eta(a) & =x(t)-x(a)+\left[\Lambda^{n}(x \mid s)\right]_{a}^{t}
\end{aligned}
$$$$
\text { on } 0 \leqq t \leqq a \text {, }
$$

where 
$\left[\Lambda^{n}(x \mid s)\right]_{a}^{t}=\Lambda^{n}(x \mid t)-\Lambda^{n}(x \mid a)=\int_{a}^{t} F^{n}\left[s, \Lambda^{0}(x \mid s), \cdots, \Lambda^{n-1}(x \mid s)\right] d s$.

Proof. We define a sequence of continuous functions $\left\{x_{p}(t)\right\}$ on $0 \leqq t \leqq 1$ by

$$
\begin{aligned}
& x_{0}(t)=\xi(t), \quad \text { on } 0 \leqq t \leqq a, \quad x_{0}(t)=\xi(a) \quad \text { on } a \leqq t \leqq 1 . \\
& x_{p}(t)=\xi(t) \quad \text { on } 0 \leqq t \leqq a,(p=1,2, \cdots) \text {, } \\
& x_{p}(t)=x_{p}(a)+\eta(t)-\eta(a)-\left[\Lambda\left(x_{p-1} \mid s\right)\right]_{a}^{t} \\
& \text { on } a \leqq t \leqq 1,(p=1,2, \cdots) \text {. }
\end{aligned}
$$

To prove that $\left\{x_{p}(t)\right\}$ converges uniformly on $a \leqq t \leqq b$ for some $b$ on $a<b \leqq 1$, we prove that for a suitably chosen $b$ in the above interval

$$
\left|x_{p+1}(t)-x_{p}(t)\right| \leqq \frac{B_{n}^{p} t^{p} M}{p !}, \quad \text { for } a \leqq t \leqq b,(p=0,1,2, \cdots),
$$

where $B_{n}, M$ are non-negative numbers.

To do this let $U$ be an arbitrary positive number satisfying $U>|\xi(a)|$ and let $b_{1}$ be such that $a<b_{1} \leqq 1$. Then according to Lemma 5 , there exists a constant $B_{n}$ such that

$$
\left|\Lambda^{n}\left(x_{l} \mid t\right)-\Lambda^{n}\left(x_{m} \mid t\right)\right| \leqq B_{n} \int_{a}^{t}\left|x_{l}(s)-x_{m}(s)\right| d s
$$

for all $t$ on $a \leqq t \leqq b_{1}$ and any pair $x_{l}, x_{m} \in \mathfrak{M}\left(a, b_{1}, \xi, U\right)$. Let $\beta$ be a variable with the domain $a \leqq \beta \leqq b_{1}$ and consider the continuous function of $\beta, \phi(\beta)$ $=\max _{a \leqq t \leqq \beta}\left|x_{1}(t)-\xi(a)\right|$. From the continuity of $x_{1}(t)$ on $I$ and from $x_{1}(a)$ $=\xi(a)$, we have $\lim _{\beta \rightarrow a+} \phi(\beta)=0$. On the other hand since $U>|\xi(a)|$, we have $\{U-|\xi(a)|\} / \exp \left\{B_{n}\right\}>0$. Therefore there is a number $b$ such that $a<b \leqq b_{1}$ and

$$
M \equiv \phi(b) \leqq\{U-|\xi(a)|\} / \exp \left\{B_{n}\right\} .
$$

With this number $b$ consider $\mathfrak{M}(a, b, \xi, U)$. By Remark 3, the inequality (4.10) holds with same $B_{n}$ for all $t$ on $a \leqq t \leqq b$ and any pair $x_{l}, x_{m} \in \mathfrak{M}(a, b, \xi, U)$. Now we prove (4.9) by complete induction on $p$. When $p=0,(4.9)$ holds by (4.7) and the definition of $M$. Assume that (4.9) holds for $p \leqq q$. Then for any $p \leqq q$, by $(4.11)$

$$
\left|x_{p+1}(t)-\xi(a)\right| \leqq M \sum_{k=0}^{p} \frac{B_{n}^{k} t^{k}}{k !} \leqq M \exp \left\{B_{n}\right\} \leqq U-|\xi(a)|, \quad \text { for } a \leqq t \leqq b
$$

so that $\left|x_{p+1}(t)\right| \leqq U$ for $a \leqq t \leqq b$ and in particular for $p=q-1, q,\left|x_{q}(t)\right|$, $\left|x_{q+1}(t)\right| \leqq U$ for $a \leqq t \leqq b$ and hence $x_{q}, x_{q+1} \in \mathfrak{M}(a, b, \xi, U)$. Then for $p=q+1$, 
by (4.8), (4.10) and the induction hypothesis, (4.9) holds. By complete induction on $p,(4.9)$ is now established. Then by Cauchy's criterion, $x(t)$ $=\lim _{p \rightarrow \infty} x_{p}(t)$ exists uniformly on $a \leqq t \leqq b$. By the definition of $\left\{x_{p}(t)\right\}, x(t)$ is continuous on $0 \leqq t \leqq 1$ and $x(t)=\xi(t)$ on $0 \leqq t \leqq a$. Also by (4.8)

$$
x(t)=\lim _{p \rightarrow \infty} x_{p}(t)=x(a)+\eta(t)-\eta(a)-\left[\Lambda^{n}(x \mid s)\right]_{a}^{t} \quad \text { on } a \leqq t \leqq b,
$$

where the passing to the limit of $p$ under the $n-1$ integral signs involved in $\Lambda^{n}(x \mid t)$ is justified by the uniform convergence of $\left\{x_{p}(t)\right\}$ and the continuity of $F^{1}, F^{2}, \cdots, F^{n}$ according to Lemma 2.

4.3. Lemma 7. Let $F^{1}, F^{2}, \cdots, F^{n}$ and their first derivatives with respect to $u, v_{1}, \cdots, v_{n-1}$ be continuous on $I \otimes R_{k}(k=1,2, \cdots, n)$. Then for every $y \in C_{w}$, the Volterra functional equation (2.3) either has a solution $x \in C_{w}$ or else it is satisfied by a function $x_{\infty}(t)$ which is defined and continuous on some interval $0 \leqq t<t_{\infty}$ with $0<t_{\infty} \leqq 1$, vanishes at $t=0$, and becomes unbounded as $t$ approaches $t_{\infty}$ from the left.

Proof. Let $y \in C_{w}$ be given. Suppose there does not exist an element of $C_{w}$ that satisfies (2.3) on $I$ for the given $y$. We prove the lemma by constructing $x_{\infty}(t)$ by applying Lemma 6 . We shall construct a solution on an interval $\left[0, t_{1}\right]$ with $0<t_{1} \leqq 1$ and then extend this solution on an interval $\left[0, t_{2}\right]$ with $t_{1}<t_{2} \leqq 1$ and so forth. Since the number $b$ in Lemma 6 is not unique, the choice of $t_{1}, t_{2}, \cdots$ at each step will not be unique. We shall use the superscript $\alpha$ for a sequence of choices of $t_{1}, t_{2}$. . .

Now let $a=0$ and $\xi(t)$ be defined on the closed interval $t=0$ by $\xi(0)=0$ and let $\eta(t)=y(t)$ on $0 \leqq t \leqq 1$. By Lemma 6 , there exists a function $x_{1}^{\alpha}(t)$ which is continuous on some interval $0 \leqq t \leqq t_{1}^{\alpha}$ with $0<t_{1}^{\alpha} \leqq 1$ and satisfies

$$
x_{1}^{\alpha}(0)=0, \quad y(t)=x_{1}^{\alpha}(t)+\left[\Lambda^{n}\left(x_{1}^{\alpha} \mid s\right)\right]_{0}^{t} \quad \text { on } 0 \leqq t \leqq \underset{t_{1}^{\alpha}}{\alpha} .
$$

We apply Lemma 6 again by setting $a=t_{1}^{\alpha}, \xi(t)=x_{1}^{\alpha}(t)$ on $0 \leqq t \leqq t_{1}^{\alpha}$ and $\eta(t)$ $=y(t)$ on $t_{1}^{\alpha} \leqq t \leqq 1$, and obtain a function $x_{2}^{\alpha}(t)$ which is continuous on some interval $0 \leqq t \leqq t_{2}^{\alpha}$ with $t_{1}^{\alpha}<t_{2}^{\alpha} \leqq 1$ and satisfies

$$
x_{2}^{\alpha}(t)=x_{1}^{\alpha}(t) \quad \text { on } 0 \leqq t \leqq t_{1}^{\alpha},
$$

and

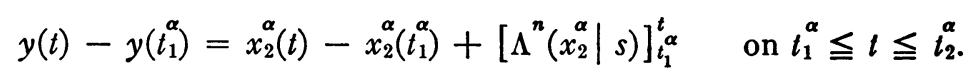

We repeat the application of Lemma 6 . This process will not be terminated since $t=1$ will never be reached as we shall see below. Now, in general $x_{m}^{\alpha}(t)$ is continuous on $\left[0, t_{m}^{\alpha}\right]$ with $0<t_{1}^{\alpha}<t_{2}^{\alpha}<\cdots<t_{m}^{\alpha} \leqq 1$, vanishes at $x=0$ and satisfies 


$$
\begin{array}{rrr}
x_{1}(0)=0, & x_{k}(t)=x_{k-1}(t) \quad \text { on }\left[0, t_{k-1}\right] \text { for } k=2,3, \cdots, m . \\
y(t)=x_{1}^{\alpha}(t)+\left[\Lambda^{n}\left(x_{1}^{\alpha} \mid s\right)\right]_{0}^{t} & \text { on }\left[0, t_{1}^{\alpha}\right], \\
y(t)-y\left(t_{k-1}^{\alpha}\right)=x_{k}^{\alpha}(t)-x_{k}^{\alpha}\left(t_{k-1}^{\alpha}\right)+\left[\Lambda^{n}\left(x_{k}^{\alpha} \mid s\right)\right]_{t_{k-1}}^{t_{\alpha}} & \\
& \text { on }\left[t_{k-1}^{\alpha}, t_{k}^{\alpha}\right] \text { for } k=2,3, \cdots, m .
\end{array}
$$

To show that $x_{m}^{\alpha}(t)$ satisfies $(2.3)$ on $\left[0, t_{m}^{\alpha}\right]$ we set, for any $k \leqq m, t=t_{1}, t_{2}, \cdots$, $t_{k-1}$ respectively in the first $k-1$ equations of (4.13) and add these $k-1$ equations and the $k$ th equation of (4.13) side by side. Thus we see that $x_{k}^{\alpha}(t)$ satisfies (2.3) on $\left[t_{k-1}^{\alpha}, t_{k}^{\alpha}\right]$ for $k \leqq m$ and then, since $x_{m}(t)=x_{k}(t)$ on $0 \leqq t \leqq t_{k}$ for $k \leqq m, x_{m}^{\alpha}(t)$ satisfies $(2.3)$ on $\left[0, t_{m}^{\alpha}\right]$. From this it follows also that $t_{m}^{\alpha} \neq 1$ for any positive integer $m$ and the sequence $\left\{x_{m}^{\alpha}(t)\right\}$ is an infinite sequence. Now since $0<t_{1}^{\alpha}<t_{2}^{\alpha}<\cdots<1$, let $t_{\infty}^{\alpha}=\lim _{m \rightarrow \infty} t_{m}^{\alpha} \leqq 1$. We now construct a function $x_{\infty}^{\alpha}(t)$ which is continuous on $\left[0, t_{\infty}^{\alpha}\right)$, vanishes at $t=0$ and satisfies (2.3) on $\left[0, t_{\infty}^{\alpha}\right)$. For any $\tau \in\left[0, t_{\infty}^{\alpha}\right)$, there is a positive integer $M$ such that $t_{M-1}^{\alpha}<\tau \leqq t_{M}^{\alpha}$. Then $x_{M}^{\alpha}(\tau)=x_{M+1}^{\alpha}(\tau)=x_{M+2}^{\alpha}(\tau)=\cdots$. Let $x_{\infty}^{\alpha}(\tau)$ be this value which is independent of $m$ for $m \geqq M$. Then a function $x_{\infty}^{\alpha}(t)$ is uniquely defined on $\left[0, t_{\infty}^{\alpha}\right)$. Since $x_{\infty}^{\alpha}(t)=x_{m}^{\alpha}(t)$ on $\left[0, t_{m}^{\alpha}\right]$ for all $m, x_{\infty}^{\alpha}(t)$ is continuous on $\left[0, t_{\infty}^{\alpha}\right)$, vanishes at $t=0$, and satisfies $(2.3)$ on $\left[0, t_{\infty}^{\alpha}\right)$.

Now consider the collection $\left\{x_{\infty}^{\alpha}(t)\right\}$ each of which is constructed in the manner described above. Since $t_{\infty}^{\alpha} \leqq 1$ for all $\alpha$, let $t_{\infty} \equiv \sup _{\alpha} t_{\infty}^{\alpha} \leqq 1$. To construct a function $x_{\infty}(t)$ which is continuous on $\left[0, t_{\infty}\right)$, vanishes at $t=0$ and satisfies (2.3) on $\left[0, t_{\infty}\right)$. Let $\tau \in\left[0, t_{\infty}\right)$. Consider all values of $\alpha$ for which $\tau<t_{\infty}^{\alpha}$. For these values of $\alpha$, the functions $x_{\infty}^{\alpha}(t)$ are continuous on $[0, \tau]$, vanish at $t=0$ and satisfy $(2.3)$ on $[0, \tau]$. Then by uniqueness of solution of $(2.3)$ on $[0, \tau]$ according to Remark 1 , the value of $x_{\infty}^{\alpha}(\tau)$ is independent of $\alpha$. Let $x_{\infty}(\tau)$ be $x_{\infty}^{\alpha}(\tau)$ which is independent of $\alpha$ for such $\alpha$ that $\tau<t_{\infty}^{\alpha}$. Then a function $x_{\infty}(t)$ is uniquely defined on $\left[0, t_{\infty}\right)$. It has the required properties.

To prove that $x_{\infty}(t)$ becomes unbounded as $t$ approaches $t_{\infty}$ from the left: Assume that $x_{\infty}(t)=\Lambda^{0}\left(x_{\infty} \mid t\right)$ is bounded on $\left[0, t_{\infty}\right)$. If $\Lambda^{k}\left(x_{\infty} \mid t\right)$ is bounded on $\left[0, t_{\infty}\right)$ for $k \leqq N$, it follows from the continuity of $F^{N+1}$ on $I \otimes R_{N+1}$ that $\Lambda^{N+1}\left(x_{\infty} \mid t\right)$ is also bounded on $\left[0, t_{\infty}\right)$. By complete induction on $k$, $\Lambda^{k}\left(x_{\infty} \mid t\right),(k=0,1,2, \cdots, n)$ are all bounded on $\left[0, t_{\infty}\right)$. Let $V$ be a positive number such that

$$
\left|\Lambda^{k}\left(x_{\infty} \mid t\right)\right| \leqq V \quad \text { on } 0 \leqq t<t_{\infty} \text { for } k=0,1,2, \cdots, n .
$$

Let the sequence of functions $F^{* *}\left(t, u, v_{1}, \cdots, v_{k-1}\right),(k=1,2, \cdots, n)$ be so defined that $F^{k *}=F^{k}$ if $\left|v_{j}\right| \leqq V$ for all $j=0,1,2, \cdots, k-1$, for $k=1,2$, $\cdots, n$ and beyond the above $k$-dimensional rectangle $F^{k *}$ is continued as constant in the way $F^{k *}$ was defined in the proof of Theorem I. The sequence of functionals $\Lambda^{k *}(x \mid t),(k=0,1,2, \cdots, n)$ is constructed with $F^{k *},(k=1,2, \cdots, n)$ by $(2.0),(2.1),(2.2)$. Then $x_{\infty}(t)$ is a solution of $y(t)$ $=x(t)+\Lambda^{n *}(x \mid t)$ on $0 \leqq t<t_{\infty}$. On the other hand, this modified functional 
equation has a solution $\tilde{x} \in C_{w}$ by Lemma 3 . According to Remark $2, x_{\infty}(t)$ $=\bar{x}(t)$ on $\left[0, t_{\infty}\right)$. Let us now extend $x_{\infty}(t)$ to $t=t_{\infty}$ by defining $x_{\infty}\left(t_{\infty}\right)=\tilde{x}\left(t_{\infty}\right)$. Then $x_{\infty}(t)$ satisfies the modified functional equation on $\left[0, t_{\infty}\right]$. Now since (4.14) holds not only for $\left[0, t_{\infty}\right)$ but also for $\left[0, t_{\infty}\right], x_{\infty}(t)$ satisfies $(2.3)$ on $\left[0, t_{\infty}\right]$. Thus if $t_{\infty}=1$, we have a solution $x_{\infty} \in C_{w}$ for (2.3), which is a contradiction. But if $t_{\infty}<1$, then by applying Lemma 6 we can extend $x_{\infty}(t)$ beyond $t_{\infty}$, which contradicts the definition of $t_{\infty}$. Therefore $x_{\infty}(t)$ must become unbounded as $t$ approaches $t_{\infty}$ from the left.

4.4. Proof of Theorem II. Suppose that for some $y \in C_{w}$ there is no $x \in C_{w}$ that satisfies (2.3). Then by Lemma 7 , there is a function $x(t)$ which vanishes at $t=0$, is continuous and satisfies (2.3) on some interval $0 \leqq t<t_{\infty}$ with $0<t_{\infty} \leqq 1$ and becomes unbounded as $t$ approaches $t_{\infty}$ from the left.

If $x(t)$ is such that $x(t)>0$ on some interval $t_{0}<t<t_{\infty}$, then by (2.3), (2.2), (2.1), (2.0), (4.1), $y(t)-y\left(t_{0}\right) \geqq x(t)-x\left(t_{0}\right)-A\left(t-t_{0}\right)$ for $t_{0}<t<t_{\infty}$. Similarly, if $x(t)<0$ on $t_{0}<t<t_{\infty}$ then $y(t)-y\left(t_{0}\right) \leqq x(t)-x\left(t_{0}\right)+A\left(t-t_{0}\right)$ for $t_{0}<t<t_{\infty}$. In any case $y(t)$ becomes unbounded as $t$ approaches $t_{\infty}$ from the left. This contradicts the continuity of $y(t)$ at $t_{\infty}$.

Let us assume that for any $t_{0}$ satisfying $0<t_{0}<t_{\infty}$ there is always a point $t$ such that $t_{0}<t<t_{\infty}$ and $x(t)=0$. Let $\left\{t_{m}\right\}$ be an increasing sequence of points such that $\lim _{m \rightarrow \infty} t_{m}=t_{\infty}$ and $x\left(t_{m}\right)=0$. Let $t_{m}^{\prime}$ be so chosen that $t_{m}<t_{m}^{\prime}<t_{\infty}$ and $\left|x\left(t_{m}^{\prime}\right)\right| \geqq m$. Let $t_{m}^{\prime \prime}$ be the least upper bound of all points between $t_{m}$ and $t_{m}^{\prime}$ at which $x(t)$ vanishes. Then $x(t) \neq 0$ on $t_{m}^{\prime \prime}<t \leqq t_{m}^{\prime}$ and $x\left(t_{m}^{\prime \prime}\right)=0$ by the continuity of $x(t)$. Now if $x(t)>0$ on $t_{m}^{\prime \prime}<t \leqq t_{m}^{\prime}$ then $y\left(t_{m}^{\prime}\right) \geqq m$ and by (2.3), (2.2), (2.1), (2.0), (4.1), $y\left(t_{m}^{\prime}\right)-y\left(t_{m}^{\prime \prime}\right) \geqq m-A\left(t_{m}^{\prime}-t_{m}^{\prime \prime}\right)$. Similarly if $x(t)<0$ on $t_{m}^{\prime \prime}<t \leqq t_{m}^{\prime}$ then $y\left(t_{m}^{\prime}\right) \leqq-m$ and $y\left(t_{m}^{\prime}\right)-y\left(t_{m}^{\prime \prime}\right) \leqq-m+A\left(t_{m}^{\prime}-t_{m}^{\prime \prime}\right)$. Since $\lim _{m \rightarrow \infty} t_{m}^{\prime}=\lim _{m \rightarrow \infty} t_{m}^{\prime \prime}=t_{\infty}$, the two inequalities contradict the continuity of $y(t)$ at $t_{\infty}$. This completes the proof of Theorem II.

5. Relationship between the functional equations and the differential systems. Let $\Delta_{0}$ be the triangle $0 \leqq s \leqq t \leqq t_{0}$ and $I_{0}$ be the interval $0 \leqq t \leqq t_{0}$ and let $J^{1}(t, u), J^{2}\left(t, u, v_{1}\right), \cdots, J^{n}\left(t, u, v_{1}, \cdots, v_{n-1}\right)$ be defined and continuous on $I_{0} \otimes R_{n}$ and $y(t)$ be defined and continuous on $I_{0}$. Then let the functionals $\Omega^{k}, \Psi^{k}, \tilde{F}^{k}, \tilde{\Phi}^{k}, \tilde{\Lambda}^{k}$ be defined by

$$
\begin{gathered}
\Omega^{0}(y \mid s, t)=y(t-s) \\
\Psi^{k}\left(y \mid s, t, u, v_{1}, \cdots, v_{k-1}\right)=J^{k}\left[s, v_{0}+\Omega^{0}, \cdots, v_{k-1}+\Omega^{k-1}\right] \\
\quad \text { on } \Delta_{0} \otimes R_{k},(k=1,2, \cdots, n) . \\
\begin{array}{r}
\Omega^{k}\left(y \mid s, t, u, v_{1}, \cdots, v_{k-1}\right) \int_{s}^{t} \Psi(p) d p \quad \text { on } \Delta_{0} \otimes R_{k},(k=1,2, \cdots, n) . \\
\tilde{F}^{k}\left(t^{\prime}, u^{\prime}, v_{1}^{\prime}, \cdots, v_{k-1}^{\prime}, t, u, v_{1}, \cdots, v_{k-1}\right) \\
=t J^{k}\left[\left(1-t^{\prime}\right) t, u+t^{1 / 2} u^{\prime}, v_{1}+v_{1}^{\prime}, \cdots, v_{k-1}+v_{k-1}^{\prime}\right], \\
(k=1,2, \cdots, n) .
\end{array}
\end{gathered}
$$




$$
\begin{gathered}
\tilde{\Phi}^{k}\left(x \mid t^{\prime}, t, u, v_{1}, \cdots, v_{k-1}\right)=t \Psi^{k}\left\{t^{1 / 2} x\left[\frac{(\cdot)}{t}\right] \mid\left(1-t^{\prime}\right) t\right\}, \\
(k=1,2, \cdots, n) . \\
\tilde{\Lambda}^{k}\left(x \mid t^{\prime}, t, u, v_{1}, \cdots, v_{k-1}\right)=\Omega^{k}\left\{t^{1 / 2} x\left[\frac{(\cdot)}{t}\right] \mid\left(1-t^{\prime}\right) t\right\}, \\
\tilde{\Lambda}^{0}\left(x \mid t^{\prime}, t\right)=x\left(t^{\prime}\right) .
\end{gathered}
$$

We quote results of [1] in the following remarks.

REMARK 4. If we regard the variables $t, u, v_{1}, \cdots, v_{n-1}$ in $\tilde{F}^{k}, \tilde{\Phi}^{k}, \Lambda^{k}$ as parameters, then the recurrence relationships (2.0), (2.1), (2.2) hold for $\tilde{F}^{k}, \tilde{\Phi}^{k}, \tilde{\Lambda}^{k}$ with the variables $t^{\prime}, u^{\prime}, v_{1}^{\prime}, \cdots, v_{n-1}^{\prime}$.

REMARK 5. Let $J^{1}(t, u), J^{2}\left(t, u, v_{1}\right), \cdots, J^{n}\left(t, u, v_{1}, \cdots, v_{n-1}\right)$, $L\left(u, v_{1}, \cdots, v_{n-1}\right)$ and the first derivatives of $J^{1}, J^{2}, \cdots, J^{n-1}$ with respect to $u, v_{1}, \cdots, v_{n-2}$ be continuous on $I_{0} \otimes R_{n}$. Let $\tilde{F}^{k}, \tilde{\Phi}^{k}, \tilde{\Lambda}^{k}$ be defined by (5.4), (5.5), (5.6), (5.7) with $J^{1}, J^{2}, \cdots, J^{n}$, let $\tilde{G}$ be defined by

$$
\begin{aligned}
\widetilde{G}\left(t^{\prime}, u^{\prime}, v_{1}^{\prime}, \cdots,\right. & \left.v_{n-1}^{\prime}, t, u, v_{1}, \cdots, v_{n-1}\right) \\
& =\theta\left[\left(1-t^{\prime}\right) t, u+t^{1 / 2} u^{\prime}, v_{1}+v_{1}^{\prime}, \cdots, v_{n-1}+v_{n-1}^{\prime}\right]
\end{aligned}
$$

for $0 \leqq t^{\prime} \leqq 1$ and $v_{j}^{\prime}(j=0,1,2, \cdots, n-1)$ unrestricted, and finally, let $\tilde{g}, \tilde{f}$ be defined by

$$
\begin{array}{ll}
\text { (5.9) } \tilde{g}\left(t^{\prime}, u^{\prime}, v_{1}^{\prime}, \cdots, v_{n-1}^{\prime}\right)=-\frac{1}{2} \log \tilde{G}\left(t^{\prime}, u^{\prime}, v_{1}^{\prime}, \cdots, v_{n-1}^{\prime}\right) & \text { on } I \otimes R_{n} . \\
\text { (5.10) } \tilde{f}\left(t^{\prime}, u^{\prime}, v_{1}^{\prime}, \cdots, v_{n-1}^{\prime}\right)=\tilde{g}_{u^{\prime}} & \text { on } I \otimes R_{n} .
\end{array}
$$

Then by Remark 4 and [1, Theorem 1], the transformation

$$
y\left(t^{\prime}\right)=x\left(t^{\prime}\right)+\int_{0}^{t^{\prime}} \tilde{f}\left[s^{\prime}, \tilde{\Lambda}^{0}\left(s^{\prime}\right), \cdots, \tilde{\Lambda}^{n-1}\left(s^{\prime}\right)\right] d s^{\prime}, \quad t^{\prime} \in I,
$$

maps $C_{w}$ in a 1-1 manner into a Wiener measurable subset $\Gamma$ of $C_{w}$. Furthermore if there exists a positive solution $\theta$ of (1.1), (1.2) such that all its first derivatives and the first derivatives of $\theta_{u}$ with respect to $v_{1}, v_{2}, \cdots, v_{n-1}$ are continuous on $I_{0} \otimes R_{n}$, then

$$
\theta\left(t, u, v_{1}, \cdots, v_{n-1}\right) m_{w}(\Gamma)
$$

$$
=\int_{C_{w}} \exp \left\{\Omega^{n}(0)\right\} L\left[v_{0}+\Omega^{0}(0), \cdots, v_{n-1}+\Omega^{n-1}(0)\right] d_{w} y
$$

on $I_{0} \otimes R_{n}$. 
6. Minimality theorems. 6.1. A minimality theorem derived from Theorem $\mathrm{I}$.

TheOREM III. Let $J^{1}, J^{2}, \cdots, J^{n}, L$ and the first derivatives of $J^{1}, J^{2}, \cdots$, $J^{n-1}$ with respect to $u, v_{1}, \cdots, v_{n-2}$ be continuous on $I_{0} \otimes R_{n}$. Let $J^{1}, J^{2}, \cdots$, $J^{n-1}$ satisfy the order of growth conditions

$$
\begin{aligned}
& \left|J^{k}\left(t, u, v_{1}, \cdots, v_{k-1}\right)\right| \\
& \leqq A\left\{1+\sum_{j=0}^{k-1}\left|v_{j}\right|\right\} \log \left\{2+\sum_{j=0}^{k-1}\left|v_{j}\right|\right\} \begin{array}{r}
\text { on } I_{0} \otimes R_{k}, \\
(k=1,2, \cdots, n-1)
\end{array}
\end{aligned}
$$

where $A$ is a positive number. Suppose that there exists a positive solution $\theta$ of (1.1), (1.2) such that all its first derivatives and the first derivatives of $\theta_{u}$ with respect to $v_{1}, v_{2}, \cdots, v_{n-1}$ are continuous on $I_{0} \otimes R_{n}$ and furthermore

$$
\begin{aligned}
\frac{\left|\theta_{u}\left(t, u, v_{1}, \cdots, v_{n-1}\right)\right|}{\theta\left(t, u, v_{1}, \cdots, v_{n-1}\right)} \leqq A\left\{1+\sum_{j=0}^{n-1}\left|v_{j}\right|\right\} \log \left\{2+\sum_{j=0}^{n-1}\left|v_{j}\right|\right\} & \text { on } I_{0} \otimes R_{n},
\end{aligned}
$$

then it follows that $\theta$ is the minimal positive solution with continuous first derivatives of (1.1), (1.2) on $I_{0} \otimes R_{n}$.

Proof. We prove that for any given point $\left(t, u, v_{1}, \cdots, v_{n-1}\right) \in I_{0} \otimes R_{n}$, $m_{w}(\Gamma)=1$ in (5.12). Let $A^{\prime} \geqq \max \left\{1, \log \left(1+\left(t_{0}\right)^{1 / 2}\right)\right\}$. Then from (5.4), (6.1)

$$
\begin{aligned}
\left|\tilde{F}^{k}\left(t^{\prime}, u^{\prime}, v_{1}^{\prime}, \cdots, v_{k-1}^{\prime}, t, u, v_{1}, \cdots, v_{k-1}\right)\right| & \leqq \\
\leqq & t_{0}\left(1+\left(t_{0}\right)^{1 / 2}\right) A\left\{1+\sum_{j=0}^{k-1}\left|v_{j}\right|+\sum_{j=0}^{k-1}\left|v_{j}^{\prime}\right|\right\} \\
& \cdot \log \left\{\left(1+\left(t_{0}\right)^{1 / 2}\right)\left(2+\sum_{j=0}^{k-1}\left|v_{j}\right|+\sum_{j=0}^{k-1}\left|v_{j}^{\prime}\right|\right)\right\} \\
\leqq & 2 t_{0}\left(1+\left(t_{0}\right)^{1 / 2}\right) A A^{\prime}\left\{1+\sum_{j=0}^{k-1}\left|v_{j}\right|+\sum_{j=0}^{k-1}\left|v_{j}^{\prime}\right|\right\} \\
& \cdot \log \left\{2+\sum_{j=0}^{k-1}\left|v_{j}\right|+\sum_{j=0}^{k-1}\left|v_{j}^{\prime}\right|\right\}, \quad(k=1,2, \cdots, n-1) .
\end{aligned}
$$

Let us remark that for any two real numbers $a$ and $x$

$$
\begin{aligned}
(1+|a| & +|x|) \log (2+|a|+|x|) \\
& <4(1+|x|) \log (2+|x|)+(1+2|a|) \log (2+2|a|) \\
& <4[1+(1+2|a|) \log (2+2|a|)](1+|x|) \log (2+|x|)
\end{aligned}
$$

Then 


$$
\begin{array}{r}
\left|\tilde{F}^{k}\right| \leqq \\
\left\{t_{0}\left(1+\left(t_{0}\right)^{1 / 2}\right) A A^{\prime} A_{k}^{\prime \prime}\left(u, v_{1}, \cdots, v_{k-1}\right)\right. \\
\left\{1+\sum_{j=0}^{k-1}\left|v_{j}^{\prime}\right|\right\} \log \left\{2+\sum_{j=0}^{k-1}\left|v_{j}^{\prime}\right|\right\}
\end{array}
$$

where

$$
\begin{array}{r}
A_{k}^{\prime \prime}\left(u, v_{1}, \cdots, v_{k-1}\right)=1+\left(1+2 \sum_{j=0}^{k-1}\left|v_{j}\right|\right) \log \left(2+2 \sum_{j=0}^{k-1}\left|v_{j}\right|\right) \\
(k=1,2, \cdots, n-1) .
\end{array}
$$

Also from (5.10), (5.9), (5.8)

$$
\tilde{f}=-\frac{1}{2} \frac{t^{1 / 2} \theta_{u}\left[\left(1-t^{\prime}\right) t, u+t^{1 / 2} u^{\prime}, v_{1}+v_{1}^{\prime}, \cdots, v_{n-1}+v_{n-1}^{\prime}\right]}{\theta\left[\left(1-t^{\prime}\right) t, u+t^{1 / 2} u^{\prime}, v_{1}+v_{1}^{\prime}, \cdots, v_{n-1}+v_{n-1}^{\prime}\right]}
$$

and by $(6.2),(6.3)$

$$
\begin{aligned}
\mid \tilde{f}\left(t^{\prime}, u^{\prime}, v_{1}^{\prime}, \cdots, v_{n-1}^{\prime}, t,\right. & \left.u, v_{1}, \cdots, v_{n-1}\right) \mid \\
\leqq & 4\left(t_{0}\right)^{1 / 2}\left(1+\left(t_{0}\right)^{1 / 2}\right) A A^{\prime} A_{n}^{\prime \prime}\left(u, v_{1}, \cdots, v_{n-1}\right) \\
& \cdot\left\{1+\sum_{j=0}^{n-1}\left|v_{j}^{\prime}\right|\right\} \log \left\{2+\sum_{j=0}^{n-1}\left|v_{j}^{\prime}\right|\right\}
\end{aligned}
$$

where $A_{n}^{\prime \prime}\left(u, v_{1}, \cdots, v_{n-1}\right)$ is defined by (6.5) with $k=n$.

By (6.4), (6.7), the functions $\widetilde{F}^{k}, \tilde{f}^{k}$ of the variables $t^{\prime}, u^{\prime}, v_{1}^{\prime}, \cdots, v_{n-1}^{\prime}$ satisfy the conditions of Theorem I for any given parametric values $t, u, v_{1}$, $\cdots, v_{n-1}$ and according to the conclusion of Theorem I, the transformation (5.11) maps $C_{w}$ in a 1-1 manner onto itself so that $m_{w}(\Gamma)=1$ in (5.12). Since $t, u, v_{1}, \cdots, v_{n-1}$ are arbitrarily given, $m_{w}(\Gamma)=1$ holds on $I_{0} \otimes R_{n}$. By $[1$, Theorem 4$]$, the proof is completed.

\subsection{A minimality theorem derived from Theorem II.}

Lemma 8. Under the hypothesis of Remark 5 on $J^{k}, L$ and $\theta$, assume further that there are numbers $t^{*} \in I_{0}$ and $u^{*} \in R_{1}$ such that for some constant $A$

$$
\frac{\theta_{u}\left[t, u^{*}+\Delta u, v_{1}, \cdots, v_{n-1}\right]}{\theta\left[t, u^{*}+\Delta u, v_{1}, \cdots, v_{n-1}\right]} \operatorname{sgn}[\Delta u] \leqq A
$$

holds for $0 \leqq t \leqq t^{*}, \Delta u$ and $v_{j}(j=1,2, \cdots, n-1)$ unrestricted, then $m_{w}(\Gamma)=1$ in (5.12) on the set $\left[0, t^{*}\right] \otimes\left\{u^{*}\right\} \otimes R_{n-1}$.

Proof. For $0 \leqq t \leqq t^{*}, \Delta u$ and $v_{j}(j=1,2, \cdots, n-1)$ unrestricted, we have from (6.6), (6.8)

$$
\tilde{f}\left(t^{\prime}, u^{\prime}, v_{1}^{\prime}, \cdots, v_{n-1}^{\prime}, t, u^{*}, v_{1}, \cdots, v_{n-1}\right) \operatorname{sgn} u^{\prime} \geqq-\frac{1}{2}\left(t^{*}\right)^{1 / 2} A
$$

for $\left(t^{\prime}, u^{\prime}, v_{1}^{\prime}, \cdots, v_{n-1}^{\prime}\right) \in I \otimes R_{n}$. Then for the parametric values in the set 
$\left[0, t^{*}\right] \otimes\left\{u^{*}\right\} \otimes R_{n-1}$, the functions $\widetilde{F}^{1}, \tilde{F}^{2}, \cdots, \tilde{F}^{n-1}, \tilde{f}$ satisfy the conditions of Theorem II and according to its conclusion, the transformation (5.11) maps $C_{w}$ in a $1-1$ manner onto itself so that $m_{w}(\Gamma)=1$ in (5.12) on the set $\left[0, t^{*}\right] \otimes\left\{u^{*}\right\} \otimes R_{n-1}$.

Theorem IV. Let $J^{1}, J^{2}, \cdots, J^{n}, L$ and the first derivatives of $J^{1}, J^{2}, \cdots$, $J^{n-1}$ with respect to $u, v_{1}, \cdots, v_{n-2}$ be continuous on $I_{0} \otimes R_{n}$. If $n>1$, let $J^{1}, J^{2}, \cdots, J^{n-1}$ be pervasive $\left({ }^{7}\right)$ in $I_{0}$. Suppose that there exists a positive solution $\theta$ of (1.1), (1.2) such that all its first derivatives and the first derivatives of $\theta_{u}$ with respect to $v_{1}, v_{2}, \cdots, v_{n-1}$ are continuous on $I_{0} \otimes R_{n}$. If for some constant $A$,

$$
\frac{\theta_{u}\left[t, u, v_{1}, \cdots, v_{n-1}\right]}{\theta\left[t, u, v_{1}, \cdots v_{n-1}\right]} \operatorname{sgn} u \leqq A
$$

on $I_{0} \otimes R_{n}$

then $\theta$ is the minimal positive solution with continuous first derivatives of (1.1), (1.2).

Proof. If (6.9) holds then (6.8) holds with $t^{*}=t_{0}, u^{*}=0$ and by the conclusion of Lemma $8, m_{w}(\Gamma)=1$ in (5.12) on the set $I_{0} \otimes\{0\} \otimes R_{n-1}$. By $[1$, Theorem 6] the proof of the theorem is completed.

\subsection{Another minimality theorem based on Theorem II.}

Lemma 9. Under the hypothesis of Remark 5 on $J^{k}, L$ and $\theta$, assume further that there is a non-negative number $A$ such that

$$
\frac{\left|\theta_{u}\left[t, u, v_{1}, \cdots, v_{n-1}\right]\right|}{\theta\left[t, u, v_{1}, \cdots, v_{n-1}\right]} \leqq A
$$

on $I_{0} \otimes R_{n}$,

then $m_{w}(\Gamma)=1$ in (5.12) on $I_{0} \otimes R_{n}$.

Proof. If (6.10) holds on $I_{0} \otimes R_{n}$, then from (6.6)

$$
\left|\tilde{f}\left(t^{\prime}, u^{\prime}, v_{1}^{\prime}, \cdots, v_{n-1}^{\prime}, t, u, v_{1}, \cdots, v_{n-1}\right)\right| \leqq \frac{1}{2}\left(t_{0}\right)^{1 / 2} A \text { on } I \otimes R_{n},
$$

for $\left(t, u, v_{1}, \cdots, v_{n-1}\right) \in I_{0} \otimes R_{n}$. Therefore $\widetilde{F}^{1}, \widetilde{F}^{2}, \cdots, \widetilde{F}^{n-1}, \tilde{f}$ satisfy the hypothesis of Theorem II for any choice of the parametric values $t, u, v_{1}, \cdots$, $v_{n-1}$ in $I_{0} \otimes R_{n}$ and by the conclusion of the theorem, the transformation (5.11) maps $C_{w}$ in a 1-1 manner onto itself so that $m_{w}(\Gamma)=1$ in (5.12) on $I_{0} \otimes R_{n}$.

Theorem V. Let $J^{1}, J^{2}, \cdots, J^{n}, L$ and the first derivatives of $J^{1}, J^{2}, \cdots$, $J^{n-1}$ with respect to $u, v_{1}, \cdots, v_{n-2}$ be continuous in $I_{0} \otimes R_{n}$. Suppose that there exists a positive solution $\theta$ of (1.1), (1.2) such that all its first derivatives and the first derivatives of $\theta_{u}$ with respect to $v_{1}, v_{2}, \cdots, v_{n-1}$ are continuous on $I_{0} \otimes R_{n}$. Then if $\theta_{u} / \theta$ is bounded on $I_{0} \otimes R_{n}$, it follows that $\theta$ is the minimal positive solution with continuous first derivatives of (1.1), (1.2).

(7) See $[1$, p. 162]. 
Proof. By Lemma 9, $m_{w}(\Gamma)=1$ in (5.12) on $I_{0} \otimes R_{n}$. By [1, Theorem 4], the proof of the theorem is completed.

7. A uniqueness theorem for solutions of the differential system. Let the functions $H^{k}$ and the functionals $\widetilde{H}^{k}(k=1,2, \cdots)$ be defined by

(7.1) $H^{k}\left(A, B, M, \epsilon, u, v_{1}, \cdots, v_{k-1}\right)=A \exp \left\{B|u|^{2-\epsilon}\right\} \prod_{j=1}^{k-1}\left\{1+\left|v_{j}\right|\right\}^{M}$,

(7.2) $\tilde{H}^{k}\left(y \mid A, B, M, \epsilon, u, v_{1}, \cdots, v_{k-1}\right)=H^{k} \exp \left\{B \max _{\rho \in I_{0}}|y(\rho)|^{2-\epsilon}\right\}$

where $A, B, M>0,2>\epsilon>0, y(\rho)$ is continuous in $\rho \in I_{0}$ and it is understood that when $k=1$, the product $\prod_{j=1}^{k-1}$ is equal to 1 . The notational convention for order of growth conditions involving $H^{k}$ is that $J^{k}\left(t, u, v_{1}, \cdots, v_{k-1}\right)$ $\leqq H^{k}(A, B, M, \epsilon)$ would mean that $J^{k}$ is bounded by a function $H^{k}$ of the type (7.1) with the specific constants $A, B, M, \epsilon$ on $I_{0} \otimes R_{n}$ whereas $J^{k}\left(t, u, v_{1}, \cdots, v_{k-1}\right) \leqq H^{k}$ would mean that $J^{k}$ is bounded on $I_{0} \otimes R_{n}$ by a function $H^{k}$ of the type (7.1) with unspecified constants.

For a function $L\left(u, v_{1}, \cdots, v_{n-1}\right)$ defined on $R_{n}$, let its "translation transform" $T_{t}^{0} L$ be defined by

$$
T_{t}^{s} L=\int_{C_{w}[0, t-s]} \exp \left\{\Omega^{n}\right\} L\left[v_{0}+\Omega^{0}, \cdots, v_{n-1}+\Omega^{n-1}\right] d_{w} y 0 \leqq s \leqq t \leqq \iota_{0},
$$

provided it exists, and for $t=s$

$$
T_{8}^{8} L=L,
$$

for $s \in I_{0}$.

$T_{t}^{s} L$ defined in this fashion with given $J^{1}, J^{2}, \cdots, J^{n}$ by (5.1), (5.2), (5.3) is a function defined on $I_{0} \otimes R_{n}$.

We quote [1, Theorem 8 ] on which our uniqueness theorem, Theorem VI, will be based.

THEOREM 8 of [1]. Let $J^{1}, J^{2}, \cdots, J^{n}, L$ and their first and second derivatives with respect to $u, v_{1}, \cdots, v_{n-1}$ (except possibly for $J_{u u}^{k}$ ) be continuous and satisfy in $I_{0} \otimes R_{n}$ the order of growth conditions

$$
\begin{gathered}
\left|J^{k}\right| \leqq H^{k},\left|J_{j}^{k}\right| \leqq H^{k},(k=1,2, \cdots, n),(j=0,1, \cdots, n-1), \\
\left|J_{i j}^{k}\right| \leqq H^{k}, \\
(k=1,2, \cdots, n),(i=1,2, \cdots, n-1), \\
J^{n} \leqq \log H^{n} .
\end{gathered}
$$

Then the function $\theta\left(t, u, v_{1}, \cdots, v_{n-1}\right)$ defined by $\theta=T_{t}^{0} L$ is a solution of the differential system (1.1), (1.2) and has all its first derivatives continuous on 
$I_{0} \otimes R_{n}$. Moreover if $L>0$ throughout $R_{n}, \theta$ is the minimal positive solution with continuous first derivatives throughout $I_{0} \otimes R_{n}$.

REMARK 6. It is proved on $\left[1\right.$, p. 167] that if $J^{1}, J^{2}, \cdots, J^{n}$ and their first derivatives are continuous and satisfy (7.5), then

$$
\left|\Psi^{k}\right| \leqq \widetilde{H}^{k}, \quad\left|\Omega^{k}\right| \leqq \widetilde{H}^{k}, \quad(k=1,2, \cdots, n) .
$$

7.1. Theorem VI. Let $J^{1}, J^{2}, \cdots, J^{n}$ and their first and second derivatives with respect to $u, v_{1}, \cdots, v_{n-1}$ (except possibly for $\left.J_{u u}^{k}\right)$ be continuous and satisfy on $I_{0} \otimes R_{n}$ the order of growth conditions (7.5) and let $J^{n}$ further satisfy

$$
\left|J^{n}\right| \leqq \log H^{n}
$$

on $I_{0} \otimes R_{n}$.

Then if $\theta$ is any solution of the differential system (1.1), (1.2) with continuous first derivatives on $I_{0} \otimes R_{n}$ such that

$$
|\theta| \leqq H^{n}
$$

on $I_{0} \otimes R_{n}$

it follows that $\theta$ is unique.

The proof of the theorem is based on the following lemma.

Lemma 10. Let $J^{1}, J^{2}, \cdots, J^{n}$ and their first and second derivatives with respect to $u, v_{1}, \cdots, v_{n-1}$ (except possibly for $J_{u u}^{k}$ ) be continuous and satisfy on $I_{0} \otimes R_{n}$

$$
\begin{array}{rr}
\left|J^{k}\right| \leqq H^{k}(A, B, M, \epsilon), & (k=1,2, \cdots, n), \\
\left|J_{j}^{k}\right| \leqq H^{k}(A, B, M, \epsilon), & (k=1,2, \cdots, n),(j=0,1, \cdots, n-1), \\
\left|J_{i j}^{k}\right| \leqq H^{k}(A, B, M, \epsilon), \quad(k=1,2, \cdots, n),(i=1,2, \cdots, n-1), & (j=0,1, \cdots, n-1) .
\end{array}
$$

where $A, B, M, \epsilon$ are such that (7.8) holds with them and besides $A \geqq 1$, and let

$$
L^{*}\left(u, v_{1}, \cdots, v_{n-1} ; M_{1}, M_{2}, \cdots, M_{n-1}\right)
$$

$$
=A \exp \left\{B_{0}\left(1+u^{2}\right)^{1-\epsilon / 6}\right\} \prod_{j=1}^{n-1}\left\{2+v_{j}{ }^{2}\right\}^{M},
$$

where $B_{0}$ is a sufficiently large positive number that satisfies

$$
B_{0}\left(1+u^{2}\right)^{1-\epsilon / 6} \geqq B(1+|u|)^{2-\epsilon / 2} \quad \text { on } u \in R_{1}
$$

and $M_{j}(j=1,2, \cdots, n-1)$ are parameters with positive values. Then the function $\theta^{*}=T_{t}^{0} L^{*}$ defined by (7.3), (7.4) is the minimal positive solution with continuous first derivatives on $I_{0} \otimes R_{n}$ of the differential system consisting of the differential equation (1.1) and the boundary condition

$$
\theta^{*}\left(0, u, v_{1}, \cdots, v_{n-1}\right)=L^{*}\left(u, v_{1}, \cdots, v_{n-1}\right)
$$


for whatever positive values of $M_{j}(j=1,2, \cdots, n-1)$. Furthermore for some values of $M_{j}(j=1,2, \cdots, n-1)$

$$
\theta^{*}>H^{n}\left(A_{0}, B, M, \epsilon\right) \text { with } A_{0}>0
$$

on $I_{0} \otimes R_{n}$

7.2. Proof of Lemma 10. $J^{1}, J^{2}, \cdots, J^{n}$ satisfy the conditions of [1, Theorem 8]. $L^{*}$ has continuous first and second derivatives with respect to $u, v_{1}, \cdots, v_{n-1}$ and, since $\left(1+u^{2}\right)^{1-\epsilon / 6} \leqq(1+|u|)^{2-\epsilon / 3}$ for $u \in R_{1}, L^{*}$ and its derivatives satisfy the order of growth conditions of [1, Theorem 8] on $L$ and its derivatives. By the conclusion of the theorem, $\theta^{*}$ is the minimal positive solution with continuous first derivatives on $I_{0} \otimes R_{n}$ of the system (1.1) and (7.15).

From (7.3), (7.13), (5.7), (5.6) we obtain for $t \in\left(0, t_{0}\right],\left(u, v_{1}, \cdots, v_{n-1}\right)$ $\in R_{n}$,

$$
\begin{aligned}
T_{t}^{0} L^{*}= & A \int_{c_{w}[0, t]} \exp \left\{\int _ { 0 } ^ { t } J ^ { n } \left[p, u+y(t-p), v_{1}+\Omega^{1}(p), \cdots, v_{n-1}\right.\right. \\
& \left.\left.+\Omega^{n-1}(p)\right] d p\right\} \\
& \cdot \exp \left\{B_{0}\left[1+[u+y(t)]^{2}\right]^{1-\epsilon / 6}\right\} \prod_{j=1}^{n-1}\left\{2+\left[v_{j}+\Omega^{\prime}(0)\right]^{2}\right\}^{M_{j}} d_{w} y .
\end{aligned}
$$

From $J^{n} \geqq-\log H^{n}$ according to (7.12), and (7.1)

$$
T_{t}^{0} L^{*} \geqq A \int_{C_{w}[0, t]} \prod_{j=1}^{5} G_{j} d_{w} y
$$

where

$$
\begin{aligned}
& G_{1}(t)=\exp \left\{-\int_{0}^{t} \log A d p\right\}, \\
& G_{2}(y \mid t, u)=\exp \left\{-B \int_{0}^{t}|u+y(t-p)|^{2-\epsilon} d p\right\} \\
& G_{3}\left(y \mid t, u, v_{1}, \cdots, v_{n-1}\right) \\
& =\exp \left\{-M \sum_{j=1}^{n-1} \int_{0}^{t} \log \left[1+\left|v_{j}+\Omega^{j}(p)\right|\right] d p\right\}, \\
& \text { (7.21) } G_{4}(y \mid t, u)=\exp \left\{B_{0}\left[1+[u+y(t)]^{2}\right]^{1-\epsilon / 6}\right\} \text {, } \\
& G_{5}\left(y \mid t, u, v_{1}, \cdots, v_{n-1}, M_{1}, \cdots, M_{n-1}\right)=\prod_{j=1}^{n-1}\left\{2+\left[v_{j}+\Omega^{j}(0)\right]^{2}\right\} M_{i} .
\end{aligned}
$$

7.3. Lower bounds for $G_{1}, G_{4}, G_{2}$.

From (7.18) and from (7.21), by our choice of $B_{0}$ according to (7.14) 


$$
\begin{aligned}
G_{1}(t) & \geqq 1 / A^{t_{0}}, \\
G_{4}(y \mid t, u) & \geqq \exp \left\{B|u+y(t)|^{2-\epsilon / 2}\right\} .
\end{aligned}
$$

As for $G_{2}$, since $|a+b|^{2-\epsilon} \leqq 4\left\{|a|^{2-\epsilon}+|b|^{2-\epsilon}\right\}$ for any two real numbers $a$ and $b$ and $0<\epsilon<2$,

(7.25) $-B \int_{0}^{t}|u+y(t-p)|^{2-\epsilon} d p \geqq-4 B t_{0}|u|^{2-\epsilon}-4 B \int_{0}^{t}|y(t-p)|^{2-\epsilon} d p$.

Let us define

$$
\|y\|_{\beta}=\max _{0 \leqq \rho \leqq \beta}|y(\rho)|
$$

Then

$$
-4 B \int_{0}^{t}|y(t-p)|^{2-\epsilon} d p \geqq-4 B t_{0}\left|\|y \mid\|_{t}^{2-\epsilon},\right.
$$

and by (7.19), (7.25), (7.27)

$$
G_{2}(y \mid t, u) \geqq \exp \left\{-4 B t_{0}|u|^{2-\epsilon}\right\} \exp \left\{-4 B t_{0}\left|\|y \mid\|_{t}^{2-\epsilon}\right\} .\right.
$$

7.4. A lower bound for $G_{3}$. For any two real numbers $a$ and $b, 1+|a+b|$ $\leqq(1+|a|)(1+|b|)$ so that

$$
-\int_{0}^{t} \log \left[1+\left|v_{j}+\Omega^{j}(p)\right|\right] d p
$$

$$
\geqq \log \left[1+\left|v_{j}\right|\right]^{-t_{0}}-\int_{0}^{t} \log \left[1+\left|\Omega^{j}(p)\right|\right] d p .
$$

Now according to our choice of $A, B, M, \epsilon$ in the statement of Lemma 10

$$
\left|\Omega^{j}\right| \leqq H^{j}(A, B, M, \epsilon) \exp \left\{B\left|\|y \mid\|_{t}^{2-\epsilon}\right\}, \quad \text { for } y \in C_{w}[0, t] .\right.
$$

Then since $A \geqq 1, B>0$, the right hand of (7.30) is not less than 1 , and from the fact that $\log (1+a) \leqq \log 2+\log a$ for $a \geqq 1$ we have

$$
\begin{aligned}
-\int_{0}^{t} \log \left[1+\left|\Omega^{j}(p)\right|\right] d p & \\
& \geqq-t_{0} \log 2-\log \left[H^{j}(A, B, M, \epsilon)\right]^{t_{0}}-t_{0} B \mid\|y\|_{t}^{2-\epsilon} .
\end{aligned}
$$

From (7.20), (7.29), (7.31),

$$
G_{3}\left(y \mid t, u, v_{1}, \cdots, v_{n-1}\right)
$$

$(7.32) \geqq \prod_{j=1}^{n-1}\left[1+\left|v_{j}\right|\right]^{-M t_{0}} \exp \left\{-(n-1) M t_{0} \log 2\right\} \prod_{j=1}^{n-1}\left[H^{j}(A, B, M, \epsilon)\right]^{-M t_{0}}$

$$
\cdot \exp \left\{-(n-1) M t_{0} B\|\| y \|_{t}^{2-\epsilon}\right\} \text {. }
$$


7.5. A lower bound for $G_{5}$. For any two real numbers $a$ and $b, 2+a^{2}$ $\geqq 1+|a|$ and $1+|a+b| \geqq(1+|a|)(1+|b|)^{-1}$ hold. Thus

$$
2+\left[v_{j}+\Omega^{j}(0)\right]^{2} \geqq\left(1+\left|v_{j}\right|\right)\left(1+\left|\Omega^{j}(0)\right|\right)^{-1} .
$$

From our choice of $A, B, M, \epsilon$ as stated in Lemma 10,

$$
1+\left|\Omega^{j}(0)\right| \leqq 2 H^{j}(A, B, M, \epsilon) \exp \left\{B\left|\|y \mid\|_{t}^{2-\epsilon}\right\} .\right.
$$

From (7.33), (7.34), the fact that $M_{j}>0,(7.22)$

$$
\begin{aligned}
& G_{5}\left(y \mid t, u, v_{1}, \cdots, v_{n-1}, M_{1}, \cdots, M_{n-1}\right) \\
& \geqq \prod_{j=1}^{n-1}\left\{\left[1+\left|v_{j}\right|\right]^{M_{i} 2^{-M_{j}}}\left[H^{i}(A, B, M, \epsilon)\right]^{-M_{j}}\right\} \\
& \cdot \exp \left\{-B \sum_{j=1}^{n-1} M_{j}\|y\|_{t}^{2-\epsilon}\right\} .
\end{aligned}
$$

7.6. A lower bound for $T_{t}^{0} L^{*}$. Substituting (7.23), (7.28), (7.32), (7.24), (7.35) in (7.17), we have

$$
T_{t}^{0} L^{*} \geqq I \quad \text { on }\left(0, t_{0}\right] \otimes R_{n}
$$

where

$$
I=K G \int_{C_{w}[0, t]} \exp \left\{B|u+y(t)|^{2-\epsilon / 2}\right\} \Gamma d_{w} y
$$

with

$$
\begin{aligned}
& K\left(M_{1}, \cdots, M_{n-1}\right)=A^{1-t_{0}} \exp \left\{-(n-1) M t_{0} \log 2\right\} \prod_{j=0}^{n-1} 2^{-M_{j}}>0 \\
& G\left(u, v_{1}, \cdots, v_{n-1}, M_{1}, \cdots, M_{n-1}\right)
\end{aligned}
$$

$$
=\exp \left\{-4 B t_{0}|u|^{2-\epsilon}\right\} \prod_{j=1}^{n-1}\left\{\left[1+\left|v_{j}\right|\right]^{M i-M t_{0}}\left[H^{i}(A, B, M, \epsilon)\right]^{-M t_{0}-M_{i}}\right\}
$$

$$
\begin{aligned}
& \Gamma\left(y \mid M_{1}, \cdots, M_{n-1}\right)=\exp \left\{-B_{1}\left|\|y \mid\|_{t}^{2-\epsilon}\right\}\right. \\
& B_{1}=4 B t_{0}+(n-1) M t_{0} B+B \sum_{j=1}^{n-1} M_{j}>0 .
\end{aligned}
$$

So far the only condition on $M_{1}, \cdots, M_{n-1}$ has been that they be positive and as long as this condition is satisfied (7.36) holds. We now proceed to choose such positive values for $M_{1}, \cdots, M_{n-1}$ that the powers of

$$
1+\left|v_{1}\right|, \cdots, 1+\left|v_{n-1}\right| \text { in } G \text { are all equal to } M \text {. }
$$


7.7. Choice of values for the parameters $M_{1}, M_{2}, \cdots, M_{n-1}$. From (7.1), (7.39)

$$
\begin{aligned}
G\left(u, v_{1}, \cdots, v_{n-1}, M_{1}, \cdots, M_{n-1}\right) \\
=A^{-(n-1) M_{0}}\left\{\prod_{j=1}^{n-1} A^{-M_{j}}\right\} \exp \left\{-B_{1}|u|^{2-\epsilon}\right\} \\
\cdot\left\{\prod_{j=1}^{n-1}\left[1+\left|v_{j}\right|\right]^{M_{j}-M t_{0}}\right\}\left\{\prod_{j=1}^{n-1} \prod_{l=1}^{j-1}\left[1+\left|v_{l}\right|\right]^{-M^{2} t_{0}-M M_{j}}\right\} .
\end{aligned}
$$

From (7.42), it follows that if we make the choice

$$
\begin{aligned}
& M_{n-1}=M+M t_{0}, \\
& M_{n-2}=M+M t_{0}+M^{2} t_{0}+M M_{n-1}, \\
& M_{n-3}=M+M t_{0}+2 M^{2} t_{0}+M M_{n-1}+M M_{n-2}, \\
& \vdots \\
& \dot{M}_{1}=M+M t_{0}+(n-2) M^{2} t_{0}+M M_{n-1}+M M_{n-2}+\cdots+M M_{2},
\end{aligned}
$$

then $M_{1}, \cdots, M_{n-1}$ are all positive and the powers of $1+\left|v_{1}\right|, \cdots, 1+\left|v_{n-1}\right|$ in (7.42) all become $M$. From now on $M_{1}, \cdots, M_{n-1}$ will stand for these values. Now from (7.42)

$$
\begin{aligned}
& G\left(u, v_{1}, \cdots, v_{n-1}, M_{1}, \cdots, M_{n-1}\right) \\
& \quad=A^{-(n-1) M t_{0}}\left\{\prod_{j=1}^{n-1} A^{-M_{j}}\right\} \exp \left\{-B_{1}|u|^{2-\epsilon}\right\} \prod_{j=1}^{n-1}\left[1+\left|v_{j}\right|\right]^{M},
\end{aligned}
$$

and from (7.37), (7.43)

$$
\begin{aligned}
I= & K_{1} \exp \left\{-B_{1}|u|^{2-\epsilon}\right\} \prod_{j=1}^{n-1}\left[1+\left|v_{j}\right|\right]^{M} \\
& \cdot \int_{C[0, \ell]} \exp \left\{B|u+y(t)|^{2-\epsilon / 2}\right\} \Gamma(y \mid) d_{w} y
\end{aligned}
$$

where

$$
K_{1}=K A^{-(n-1) M t_{0}} \prod_{j=1}^{n-1} A^{-M_{j}}>0 .
$$

It remains to estimate the Wiener integral (7.44).

7.8. Lower bound of the Wiener integral. Let us decompose the Wiener integral $I$ given by (7.44) into two Wiener integrals $I^{+}, I^{-}$over the two intervals $C_{w}^{+}[0, t], C_{w}^{-}[0, t]$ of the Wiener space $C[0, t]$ where

$$
C_{w}^{+}[0, t]=\left\{y \in C_{w}[0, t] \mid y(t) \geqq 0\right\}, \quad C_{w}^{-}[0, t]=\left\{y \in C_{w}[0, t] \mid y(t)<0\right\},
$$


each with a measure $1 / 2$. Then

$$
I=I^{+}+I^{-}
$$

on $I_{0} \otimes R_{n}$

and from the fact that the integrand of $I$ is positive

$$
I>I^{+}, \quad I>I^{-}, \quad \text { on } I_{0} \otimes R_{n} .
$$

We now proceed to give a lower estimate for $I^{+}$when $u \geqq 0$ and a lower estimate for $I^{-}$when $u<0$. Consider $I^{+}$. Since $y(t)$ in its integrand is nonnegative, $|u+y(t)| \geqq u$ for $u \geqq 0$ and

$$
\exp \left\{B|u+y(t)|^{2-\epsilon / 2}\right\} \geqq \exp \left\{B|u|^{2-\epsilon / 2}\right\}, \quad \text { for } u \geqq 0,
$$

and consequently from (7.44)

$$
\begin{aligned}
I^{+} \geqq & K_{1} \exp \left\{-B_{1}|u|^{2-\epsilon}\right\} \\
& \cdot \exp \left\{B|u|^{2-\epsilon / 2}\right\} \prod_{j=1}^{n-1}\left[1+\left|v_{j}\right|\right]^{M} \int_{C_{w}^{+}[0, t]} \Gamma(y \mid) d_{w} y, \quad \text { for } u \geqq 0 .
\end{aligned}
$$

Since for $B, B_{1}>0$ there exists a positive constant $K_{2}$ such that

(7.49) $\exp \left\{B|u|^{2-\epsilon / 2}\right\} \exp \left\{-B_{1}|u|^{2-\epsilon}\right\} \geqq K_{2} \exp \left\{B|u|^{2-\epsilon}\right\}$, for $u \in R_{1}$, we have

(7.50) $\quad I^{+} \geqq K_{3} \exp \left\{B|u|^{2-\epsilon}\right\} \coprod_{j=1}^{n-1}\left[1+\left|v_{j}\right|\right]^{M} \int_{C_{w}^{+}\{0, t]} \Gamma(y \mid) d_{w} y$, for $u \geqq 0$ where $K_{3}=K_{1} K_{2}>0$. In estimating the Wiener integral in the right side of (7.50) we first write

$$
\int_{C_{w}^{+}[0, t]} \Gamma(y \mid) d_{w} y=\int_{C_{w}[0, t]} \frac{1}{2}\{1+\operatorname{sgn}[y(t)]\} \Gamma(y \mid) d_{w} y
$$

and then reduce the integral to one over $C_{w}[0, t]$ by using the transformation formula $\left({ }^{8}\right)$

$$
\int_{C_{w}[a, b]} F[y] d_{w} y=\int_{C_{w}[0,1]} F\left[(b-a)^{1 / 2} x\left[\frac{(\cdot)-a}{b-a}\right]\right] d_{w} x .
$$

Then

$$
\begin{aligned}
\int_{C_{w}[0, t]} \frac{1}{2}\{1+\operatorname{sgn}[y(t)]\} & \Gamma(y \mid) d_{w} y \\
& =\int_{C_{w}[0,1]} \frac{1}{2}\{1+\operatorname{sgn}[x(1)]\} \Gamma\left[t^{1 / 2} x\left(\frac{(\cdot)}{t}\right)\right] d_{w} x .
\end{aligned}
$$

From (7.40)

(8) See [3]. 


$$
\begin{aligned}
\Gamma\left[t^{1 / 2} x\left(\frac{(\cdot)}{t}\right)\right] & =\exp \left\{-B_{1}\left\|t^{1 / 2} x\left(\frac{(\cdot)}{t}\right)\right\| \|_{t}^{2-\epsilon}\right\} \\
& \geqq \exp \left\{-B_{1} t_{0}^{1-\epsilon / 2}\|x\|^{2-\epsilon}\right\}
\end{aligned}
$$

where by definition $\||x|\|=\|\| x \mid \|_{1}$. Thus

$$
\begin{aligned}
\int_{C_{w}^{+}[0, t]} \Gamma(y \mid) d_{w} y & \\
& \geqq \int_{C_{w}[0,1]} \frac{1}{2}\{1+\operatorname{sgn}[x(1)]\} \exp \left\{-B_{1} t_{0}^{1-\epsilon / 2}\|x\| \|^{2-\epsilon}\right\} d_{w} x .
\end{aligned}
$$

Since $\int_{C_{w}[0,1]} \exp \left\{B|\| x|||^{2-\epsilon}\right\} d_{w} x$ exists as a finite number for any real number $B$ and $2>\epsilon>0\left({ }^{9}\right)$ the Wiener integral in the right side of $(7.52)$ is finite. Let us call it $K_{4}$. Then from (7.50), (7.52), with $K_{5}=K_{3} K_{4}>0$

$$
I^{+} \geqq K_{5} \exp \left\{B|u|^{2-\epsilon}\right\} \prod_{j=1}^{n-1}\left[1+\left|v_{j}\right|\right]^{M}, \quad \text { for } u \geqq 0 .
$$

As for the integrand of $I^{-}$, since $y(t)$ is negative, $|u+y(t)| \geqq|u|$ for $u<0$. Following the same line of argument as for $I^{+}$we obtain

$$
I^{-} \geqq K_{5}^{\prime} \exp \left\{B|u|^{2-\epsilon}\right\} \prod_{j=1}^{n-1}\left[1+\left|v_{j}\right|\right]^{M}, \quad \text { for } u<0,
$$

where $K_{5}^{\prime}=K_{3} K_{4}^{\prime}$ and

$$
K_{4}^{\prime}=\int_{C[0,1]} \frac{1}{2}\{1-\operatorname{sgn}[x(1)]\} \exp \left\{-B_{1} t_{0}^{1-\epsilon / 2} \mid\|x\|^{2-\epsilon}\right\} d_{w} x,
$$

a finite positive number. If we now let $K_{6}=\min \left\{K_{5}, K_{6}^{\prime}\right\}$, then from (7.47), (7.53), (7.54)

$$
I>K_{6} \exp \left\{B|u|^{2-\epsilon}\right\} \prod_{j=1}^{n-1}\left[1+\left|v_{j}\right|\right]^{M} \quad \text { on }\left(0, t_{0}\right] \otimes R_{n} .
$$

From (7.36), (7.55)

$$
T_{t}^{0} L^{*} \geqq K_{6} \exp \left\{B|u|^{2-\epsilon}\right\} \prod_{j=1}^{n-1}\left[1+\left|v_{j}\right|\right]^{M} \quad \text { on }\left(0, t_{0}\right] \otimes R_{n} .
$$

If we let $A_{0}=\min \left\{A, K_{6}\right\}$ then from (7.4), (7.13), (7.14), (7.56)

$$
T_{t}^{0} L^{*} \geqq A_{0} \exp \left\{B|u|^{2-\epsilon}\right\} \prod_{j=1}^{n-1}\left[1+\left|v_{j}\right|\right]^{M} \quad \text { on } I_{0} \otimes R_{n} .
$$

This completes the proof of Lemma 10 .

(9) See [4]. 
7.9. Proof of Theorem VI. Let us assume without loss of generality that the functions $H^{k}(k=1,2, \cdots, n)$ in the hypothesis of the theorem all have the same constants $A, B, M, 2-\epsilon$ which are so large as to satisfy the condition imposed on them in the statement of Lemma 10. Then for $\theta^{*}=T_{t}^{0} L^{*}$ with $L^{*}$ defined by (7.13), the lemma applies.

Now assume that the differential system (1.1), (1.2) has two solutions $\theta_{1}, \theta_{2}$ with continuous first derivatives and satisfying (7.10) on $I_{0} \otimes R_{n}$. Let $\theta_{3}=\theta_{1}-\theta_{2}$ on $I_{0} \otimes R_{n}$. We show that $\theta_{3} \equiv 0$ on $I_{0} \otimes R_{n}$. Consider

$$
\theta_{4}=\theta^{*} \pm \frac{A_{0}}{2 A} \theta_{3}
$$

$\theta_{4}$ has continuous first derivatives and satisfies (1.1), (7.15) on $I_{0} \otimes R_{n}$. Since $\theta_{1}, \theta_{2}$ satisfy $(7.10)$, we have $\left|\theta_{3}\right| \leqq H^{n}(2 A, B, M, \epsilon)$ on $I_{0} \otimes R_{n}, A_{0}(2 A)^{-1}\left|\theta_{3}\right|$ $\leqq H^{n}\left(A_{0}, B, M, \epsilon\right)$ and by (7.57), (7.16) we have $\theta_{4}>0$ on $I_{0} \otimes R_{n}$ for both of the two signs in (7.57). Then since $\theta^{*}$ is the minimal positive solution with continuous first derivatives of (1.1), (7.15), we have $\theta_{4} \geqq \theta^{*}$ on $I_{0} \otimes R_{n}$ for both of the two signs in (7.57). This implies that $A_{0}(2 A)^{-1} \theta_{3} \equiv 0$ on $I_{0} \otimes R_{n}$ and hence $\theta_{3} \equiv 0$ on $I_{0} \otimes R_{n}$. This completes the proof of the theorem.

\section{BIBL.IOGRAPHY}

1. R. H. Cameron, Nonlinear Volterra functional equations and linear parabolic differential systems, J. Analyse Math. vol. 5 (1956/1957) pp. 136-182.

2. N. Wiener, Generalized harmonic analysis, Acta Math. vol. 55 (1930) pp. 117-258.

3. E. H. Cuthill, Integrals on spaces of functions which are real and continuous on finite and infinite intervals, Thesis, University of Minnesota, December, 1953.

4. P. Erdös and M. Kac, On certain limit theorems of the theory of probability, Bull. Amer. Math. Soc. vol. 52 (1946) pp. 292-302.

UNIVERSITY OF MINNESOTA, MinNEAPOLIS, MinNESOTA

Massachusetts Institute of Technology,

Cambridge, Massachusetts 